\title{
Processo de internação em clínica cirúrgica de um hospital público universitário
}

\author{
Process of hospitalization at surgical-clinic unit at a public university hospital \\ Proceso de hospitalización en unidad clínica quirúrgica de un hospital universitario público
}

\author{
Nadja Maria Queiroz de Albuquerquel; Alba Lucia Castelo Branco"; Maria Regina Araujo Reicherte Pimentel'"; \\ Frances Valeria Costa e Silva ${ }^{\prime V}$; Luciana Guimarães Assad ${ }^{v}$; Matheus de Albuquerque Santos ${ }^{V I}$
}

\begin{abstract}
RESUMO
Objetivo: descrever o atual processo de convocação de pacientes para procedimento cirúrgico, bem como a comunicação entre a equipe multiprofissional e o paciente no período pré-operatório e identificar a linha de cuidados prestados pela equipe multiprofissional no período pré-operatório. Método: pesquisa exploratória, descritiva e qualitativa. Amostra contemplou 28 participantes, membros da equipe multidisciplinar da clínica cirurgia geral de um hospital universitário do Rio de Janeiro. Os dados foram coletados por dois roteiros de entrevista semiestruturada, de fevereiro a abril de 2019, após autorização do Comitê de Ética em Pesquisa. Resultados: a falta de planejamento, protocolos e fluxo intenso de pacientes levou a dúvidas, estresse, cancelamento de cirurgias no decorrer da internação. Conclusão: a ausência de protocolos articulados com ações e treinamentos prejudicou o processo de internação, bem como a comunicação entre a equipe multiprofissional e paciente. Fizeram parte da linha de cuidados: anamnese, sinais vitais, conciliação medicamentosa e orientações gerais.
\end{abstract}

Descritores: Hospitalização; Cuidados de enfermagem; Comunicação; Assistência Perioperatória.

\section{ABSTRACT}

Objective: to describe the current process of calling patients in for surgical-clinic unit, as well as communication between multidisciplinary team and patient in the preoperative period. Method: in this exploratory, qualitative, descriptive study, the 28 participants were members of the multidisciplinary team of surgical-clinic unit of a university hospital in Rio de Janeiro. Data were collected from February to April 2019 using two semi-structured interview scripts. The study was authorized by the research ethics committee. Results: the lack of planning and protocols and the intense flow of patients led to doubts, stress, and cancellation of surgeries during hospitalization. Conclusion: the absence of protocols coupled with actions and training impaired the hospitalization process, as well as communication between multidisciplinary team and patient. The line of care comprised: anamnesis, vital signs, medication reconciliation and general guidelines.

Descriptors: Hospitalization; Nursing Care; Communication; Perioperative Care.

\section{RESUMEN}

Objetivo: describir el proceso actual de convocatoria de pacientes para unidad clínica quirúrgica, así como la comunicación entre equipo multidisciplinario y paciente en el período preoperatorio. Método: en este estudio exploratorio, cualitativo, descriptivo, los 28 participantes eran miembros del equipo multidisciplinario de la unidad clínica quirúrgica de un hospital universitario de Río de Janeiro. Los datos se recopilaron de febrero a abril de 2019 mediante dos guiones de entrevistas semiestructurados. El estudio fue autorizado por el comité de ética en investigación. Resultados: la falta de planificación y protocolos y el intenso flujo de pacientes generó dudas, estrés y cancelación de cirugías durante la hospitalización. Conclusión: la ausencia de protocolos sumados a acciones y formación perjudicó el proceso de internación, así como la comunicación entre equipo multidisciplinar y paciente. La línea de atención comprendió: anamnesis, constantes vitales, conciliación de medicamentos y pautas generales.

Descriptores: Hospitalización; Atención de enfermería; Comunicación; Atención Perioperativa.

\section{INTRODUÇÃO}

Os processos de trabalho nas instituições de saúde assumem configurações diversas, sendo possível admitir que alguns modos operativos possam oferecer resultados mais adequados no cuidado à saúde humana ${ }^{1}$. A partir desta referência, a observação empírica das ações que compõem o processo de internação para realização de um procedimento cirúrgico em um hospital universitário tornou-se referência para a construção deste estudo.

'Enfermeira. Especialista em CTI adulto e idoso. Universidade do Estado do Rio de Janeiro. Brasil. E-mail: nadja.qa@gmail.com. ORCID: https://orcid.org/0000-0003-2782-7684 "Enfermeira. Doutora. Professora Associada da Universidade do Estado do Rio de Janeiro. Brasil. E-mail: profalbauerj@gmail.com. ORCID: https://orcid.org/0000-0003-4055-4190 I"Enfermeira. Doutora. Professora Adjunta da Universidade do Estado do Rio de Janeiro. Brasil. E-mail: mariaregina.pimentel85@gmail.com. ORCID: https://orcid.org/0000-0002-6990-5137 IVEnfermeira. Doutora. Professora Associada. Universidade do Estado do Rio de Janeiro. Brasil. E-mail: francesvcs@gmail.com. ORCID: https://orcid.org/0000-0002-0441-2294 vEnfermeira. Doutora. Professora Associada da Universidade do Estado do Rio de Janeiro. Brasil. E-mail: Igassad@gmail.com. ORCID: https://orcid.org/0000-0003-1134-2279 VIMédico. Residente em Cirurgia Geral do Hospital Cirurgia pelo Hospital Escola da Faculdade de Medicina de Sergipe. Aracaju. Brasil. E-mail: mas.uerj@gmail.com. ORCID: https://orcid.org/0000-0003-4764-0649 
A hospitalização é uma circunstância desconfortável para o indivíduo e para a família, visto que institui alteração em seus hábitos de vida, rotinas, privacidade, além de distanciamentos familiares e de utensílios significativos. 0 procedimento cirúrgico pode representar um momento marcante na vida da pessoa, ao gerar dúvidas e vulnerabilidades, podendo prejudicar a recuperação do paciente no pós-cirúrgico ${ }^{2}$.

Nesse estudo e na prática diária, percebe-se que o processo de internação provoca ansiedade, estresse e medo da dor e anestesia no paciente. É válido salientar que não se sabe o conteúdo real da circunscrição social do paciente, de modo que é difícil estimar o quanto e como as vivências cirúrgicas influenciam o indivíduo, tornando-se necessário que, na admissão pré-operatória multidisciplinar, baseada no conhecimento técnico-científico, seja permitida ao paciente cirúrgico a compreensão de todo o processo ${ }^{3,4}$. A admissão pré-operatória contribui para identificar fatores de risco e esclarecer dúvidas do paciente e família sobre o processo cirúrgico de forma clara e objetiva, visando à humanização das ações e dos serviços de saúde ${ }^{4}$.

Nesse segmento, a Política Nacional de Regulação no Brasil, regulamentada em 2008, apresenta três diretrizes. São elas: regulação de sistemas de saúde; regulação da atenção à saúde e regulação do acesso à assistência. Tais diretrizes têm por finalidade alcançar a equidade e humanização, de modo a garantir acesso aos grupos populacionais mais necessitados de saúde, atenuando a relação da necessidade e oferta para a realização de um procedimento cirúrgico ${ }^{5,6}$.

Os procedimentos cirúrgicos têm o propósito de preservar vidas, compreendendo que este fato demanda uma atenção na promoção do atendimento seguro, em todos os níveis de assistência. Diante disso, faz-se necessária a comunicação efetiva entre a equipe multiprofissional visando reduzir a ocorrência de eventos adversos. Tais eventos são definidos como incidentes gerados pelo processo de cuidado, que repercutem em dano real e de variada magnitude à saúde do paciente. Nesse caso, a equipe precisa estar bem alicerçada de modo a reduzir danos ao paciente e, além disso, ser a luz para o cuidado integral e humanizado ${ }^{7,8}$.

Nessa perspectiva, parte-se do pressuposto que a orientação, a sensibilização e o acolhimento aos pacientes no período pré-cirúrgico podem diminuir sua insegurança e outros estressores do processo de internação. Desse modo, minimizam a ocorrência de alterações fisiológicas e contribuem para a prevenção de complicações, evidenciando o quanto a ação da equipe multiprofissional é imprescindível nesse processo, justificando assim, esta pesquisa.

O estudo teve como objetivos descrever o atual processo de convocação de pacientes para procedimento cirúrgico, bem como a comunicação entre a equipe multiprofissional e o paciente no período pré-operatório e identificar a linha de cuidados prestados pela equipe multiprofissional no período pré-operatório.

\section{MÉTODO}

Este estudo é do tipo pesquisa de campo exploratório, descritivo e de natureza qualitativa. Teve-se como cenário uma clínica de cirurgia geral de um hospital universitário, situado no município do Rio de Janeiro. A unidade de internação atualmente tem 18 leitos, sendo nove masculinos e nove femininos. A rotatividade de pacientes é alta, expressa pelo tempo médio de internação de três dias.

Foram participantes do estudo os membros da equipe multiprofissional, composta por técnicos de enfermagem, enfermeiros, residentes de medicina, nutrição e psicologia da clínica de cirurgia geral e, ainda, a funcionária administrativa do Núcleo Interno de Regulação (NIR).

O fechamento amostral ocorreu com 28 participantes, número este delineado durante o trabalho de campo, quando a organização dos dados e depoimentos apresentou a saturação dos dados, isto é, a constatação da particularidade dos achados nas entrevistas, em concordância com os temas e a veracidade dos enunciados.

Na coleta de dados, ocorrida no período de fevereiro a abril de 2019 , utilizaram-se dois instrumentos de entrevista semiestruturada, ambos com perguntas abertas referentes ao processo de internação, comunicação e condutas com o paciente e questões fechadas sobre dados socioeconômicos e demográficos. O primeiro instrumento era direcionado aos residentes médicos e composto por sete perguntas e o segundo, com cinco questões, para a equipe de residentes de enfermagem, enfermeiros e técnicos de enfermagem. As sete questões direcionadoras da entrevista eram: 1. Quais critérios ou fatores você utiliza para convocar o paciente para a internação, com vistas à realização do procedimento cirúrgico? 2. Como e quando você comunica à equipe que o paciente será internado na unidade para realizar um procedimento cirúrgico? 3. Como é sua comunicação com a equipe multiprofissional e com o paciente cirúrgico? 4 . Você recepciona o paciente no momento da internação? Em caso afirmativo, como é a recepção? Caso, não, justifique. 5. Quais condutas você implementa no período pré-operatório do paciente? 6. Você identifica que a forma que se dá o processo de internação causa alguma consequência para o paciente no período perioperatório? Qual? 7. Você tem alguma sugestão para melhorar as fases de implementação e acolhimento no processo de internação do paciente cirúrgico no período pré-operatório? 
O estudo foi realizado em concordância com a Resolução no 466/2012 ${ }^{9}$, com parecer favorável do Comitê de Ética em Pesquisa (CEP) da instituição, sob o protocolo/parecer no 3.094.455.

Os participantes foram informados sobre o processo da entrevista, previamente agendada, garantindo a autonomia, a não maleficência, a beneficência, a justiça e a equidade. Desse modo, visou-se assegurar os direitos e deveres relativos aos participantes da pesquisa. As entrevistas foram realizadas em um ambiente reservado na enfermaria de cirurgia geral, gravadas em mídia digital, armazenadas em pastas do Windows, transcritas e analisadas.

$O$ método de caracterização dos participantes utilizado foi a codificação alfanumérica com a letra $E$ para (Enfermeiros), TE para (Técnicos de Enfermagem), RM para (Residente Medicina), RN para (Residente Nutrição), RP (Residente Psicologia) e PA (Profissional Administrativo) seguida de sequência numérica aleatória, a fim de preservar o anonimato.

Foi realizada a análise de conteúdo, cumprindo as seguintes etapas: pré-análise; exploração do material ou codificação; tratamento dos resultados, inferência e interpretação ${ }^{10,11}$.

Após a leitura flutuante dos depoimentos, foram selecionadas as unidades de significação, caracterizadas por frases, resultando em 628 Unidades de Registro (UR). Estas foram agrupadas em 41 temas, divididos por assuntos semelhantes, dos quais emergiram três grandes categorias e quatro subcategorias a partir da quantificação das unidades de registro de cada tema.

\section{RESULTADOS}

A organização dos dados gerou duas dimensões de resultados. A primeira está relacionada a uma breve caracterização dos participantes. A segunda apresenta as três categorias, e suas subcategorias, e as falas dos participantes organizadas conforme os objetivos da pesquisa.

\section{Caracterização dos participantes}

Quanto aos participantes entrevistados foram observados dois grupos - um que atua como profissionais efetivos e um grupo temporário, composto por residentes multiprofissionais.

A maioria dos entrevistados foram profissionais pertencentes ao corpo permanente da instituição - 17(60,71\%). No que se refere ao sexo de ambos os grupos, $24(85,71 \%)$ foram do sexo feminino. A categoria mais presente foi a de técnicos de enfermagem, com $13(46,43 \%)$ participantes. Já no grupo de residentes, os que mais se destacaram foram os residentes de medicina, com $8(28,57 \%)$.

No tocante à faixa etária dos participantes, a idade variou entre 20 e 59 anos. A faixa etária que obteve mais evidência nos dois grupos foi a de 30 a 39 anos, com 9(32,14\%) profissionais. Quanto ao tempo de atuação profissional, foi notório o período de 1 a 7 anos - 25(89,29\%) dos entrevistados.

\section{Descrição das Categorias}

\section{Categoria 1 - Comunicação / interação}

Trata da comunicação institucional entre profissionais e pacientes e abarcou $316(50,32 \%)$ UR, sendo a mais eloquente das categorias, composta por duas subcategorias.

\section{Subcategoria 1 - Comunicação entre equipe multiprofissional e paciente no período pré-operatório}

Nesta subcategoria, foram abordados temas como a comunicação efetiva e de qualidade com o paciente; em contrapartida, houve relatos de uma comunicação deficiente entre equipe médica e paciente.

Procurar ser mais objetivo possível, orientando quanto às questões de dependência, passar as rotinas do setor de maneira clara e simples para que todos entendam (TE 01).

A gente observa que o paciente não é totalmente esclarecido; tem médico que não tem uma interação nem com a equipe nem com paciente, isso acontece aqui (...) (TE 02).

A comunicação com os pacientes; alguns médicos, na minha visão, deixam muito a desejar e o paciente fica muito perdido (E 19).

Do mesmo modo, evidencia-se que a comunicação ineficaz entre a equipe e o paciente impacta diretamente nas ações rotineiras e no acolhimento humanizado, ficando visível a identificação de alterações fisiológicas e psicológicas do paciente, conforme descrito em algumas falas.

Eu percebo é que o paciente vem despreparado para o hospital; alguns não trazem roupa nem material de higiene. Ele chega aqui pela manhã, só vai internar à tarde, só vai comer no jantar e isso prejudica o paciente com essa falta de informação (E 08). 
Traz em relação à pressão, isso aí você nota perfeitamente, aí chega aqui, a pressão tá alta, tá nervoso, sudoreico, eu digo: 'Gente, calma!' Calma que vai dar tudo certo (risos) (TE 22).

Às vezes liga hoje, tem que tá aqui hoje, causa certo estresse, dá um susto e causa uma ansiedade; eles chegam aqui meio hipertenso (TE 18).

\section{Subcategoria 2 - Comunicação entre membros da equipe multiprofissional}

Nesta subcategoria emergiram situações de comunicação deficiente, em virtude da falta de planejamento de admissão e alta, mas houve também relatos de trabalho em equipe e parceria.

É confuso às vezes, porque o médico interna e não vem fazer a prescrição do paciente, aí a gente fica embolado sem saber se vai fazer algum procedimento (TE 01).

Nem sempre eu encontro algum médico aqui, mas sempre que eu tenho alguma dúvida eu mando uma mensagem e consigo me comunicar com eles e com vocês (RN 11).

\section{Categoria 2 - Processo de internação}

Essa categoria englobou 200(31,85\%) UR e relata questões que interferem no processo de internação. Apresenta duas subcategorias, descritas a seguir.

\section{Subcategoria 1 - Processo de convocação do paciente cirúrgico}

Discorre sobre o descaso, as dificuldades e lacunas encontradas na comunicação e o impacto para o usuário.

Tem paciente que chega aqui ficam cinco, seis horas esperando, desconfortável e aí estressa, a pressão sobe, acarreta alguns problemas, principalmente por conta dessa falta de organização que os médicos têm (TE 01).

Outro dia a médica chamou o paciente seis horas da noite, ela virou para o paciente e falou assim: 'se ele não estiver aqui até as oito, ele perde'; isso ai traz um transtorno para o paciente psicologicamente (...) (E 19).

Esse paciente fica irritado, ele não sabe direito se vai operar, fica horas a fio aguardando em uma portaria e isso cai tudo aqui para a gente. 'Moça, eu tô com fome, passando mal' Por que não se organiza? Eu vejo o paciente prejudicado e perdido em todas as etapas, ele luta para conseguir a vaga, quando finalmente ele conseguiu a vaga, ele pensa que o problema está resolvido, não está (...) (PA 26).

A subcategoria demonstra ainda o processo de internação, o que determina a convocação do paciente pelos residentes médicos. Constantemente os pacientes são chamados dentro de um período inferior a 24 horas e, por causa desse processo, diversas cirurgias deixam de ser realizadas.

Tem o processo do ambulatório, do SISREG, da urgência e gravidade do quadro, quando tem maior gravidade a gente fura a fila e chama antes (...) a gente liga e eles ficam desesperados porque é no dia, tipo 'Você pode vir hoje?' É bem ruim, assim, a gente fica meio até constrangido de ligar e falar assim 'Vem agora', eu acho isso péssimo (RM 09).

Então, tem paciente que tem tipo três anos que está esperando; quando eles recebem essa ligação se desesperam, às vezes se desesperam tanto que nem vem operar. Isso já aconteceu algumas vezes, ele ficou tão nervoso!!! Ele falou, ah, não, doutor, não tenho condições de operar e aí não veio (RM 04).

\section{Subcategoria 2 - Admissão e cuidados no período pré-operatório}

Observa-se nesta subcategoria que a forma repentina de convocação do paciente para o procedimento cirúrgico prejudica seu processo de admissão. Ao mesmo tempo, há relatos de uma conduta efetiva e de qualidade nesse processo, como narrado.

A gente procura fazer breve anamnese, aquela que cabe ao técnico de enfermagem, coisa assim simples, que às vezes também passa despercebido pela quantidade de paciente que vem até nós (TE 02).

A gente faz uma anamnese mais ou menos focada no problema que ele tem e no histórico de base dele, coisa assim bem objetiva, é isso (RM 16).

Bom, primeiro avaliar as condições dele, encaixar toda a alimentação de acordo com a comorbidade, para ele ter o melhor conforto, vou evoluindo em prontuário (RN 11).

\section{Categoria 3 - Necessidades para melhoria do processo de internação}

Esta categoria reuniu $112(17,83 \%)$ UR e retrata sugestões de melhorias no processo de internação na clínica de cirurgia geral, conforme descrito.

Organizar, criar um fluxo de internação, criar uma rotina, estipular um horário, pra poder conseguir receber de uma maneira melhor o doente que tá chegando (...) (TE 01).

O ideal seria um setor do hospital para controlar essa fila de internação com uma lista que fosse de fácil acesso para todo mundo, fazer com que os nossos mapas sejam feitos antes (RM 17). 
A categoria traz ainda temas como falta de funcionário administrativo, consulta com toda a equipe multiprofissional no período pré-operatório e ausência de autonomia dos residentes médicos na gestão dos leitos cirúrgicos.

A gente não tem uma secretária, acaba fazemos bastante esse papel, a gente liga para o paciente, chama para internar, a gente é que faz tudo (RM 04).

Comunicar o paciente com antecedência, isso seria primordial, o paciente seria muito melhor assistido $e$ diminuiria custos no hospital (E 06).

Assim, acho bem errado que a gente controle a nossa fila, porque isso dá margem, e talvez isso seja o maior erro (RM 09).

Eu acho que se tivesse um enfermeiro responsável por uma consulta prévia, para orientar no pré e pósoperatório, para amenizar a dor, a angústia do paciente (E 19).

A gente precisa ter autonomia dos leitos aqui, porque outra clínica pode pegar e aí a gente fica à mercê e causa muito atraso no serviço (risos) (RM 17).

\section{DISCUSSÃo}

No atual processo de internação da cirurgia geral do hospital estudado, identificou-se que o contato com o paciente, a fim de realizar um procedimento cirúrgico, é realizado por meio de telefone particular do médico residente da especialização. A ausência de um profissional administrativo para realizar esse trabalho, a deficiência na comunicação entre a equipe multidisciplinar e paciente, a falta de planejamento, gerenciamento dos leitos e protocolos que facilitem o processo de internação são problemas que dificultam seu transcurso adequado. Além disso, levam ao cancelamento de cirurgias e a dúvidas, causando estresse ao paciente cirúrgico no decorrer da internação.

Diante do exposto, é plausível refletir, à luz da literatura, que a instituição que não promove o acolhimento ao paciente e ao familiar ocasiona dificuldades absolutas no enfrentamento desse processo. A sistematização do trabalho no ambiente hospitalar é uma estratégia que pode tanto minimizar como intensificar a inquietação do paciente no decorrer da internação ${ }^{12}$.

A qualidade no setor da saúde não se constitui em uma condição subjetiva e deve se apoiar nos princípios da eficácia, efetividade, eficiência, otimização, aceitabilidade, legitimidade e equidade. Esses pilares são amplamente aceitos por contemplar a lógica de funcionamento de recursos, organização, atividades, serviços e efeitos, sendo fundamentais por contribuírem para os sistemas de gestão e excelência hospitalar ${ }^{1}$.

A implantação de protocolos de cuidados nos ambientes hospitalares tem se tornado uma necessidade para a redução de agravos e melhoria da qualidade do atendimento, o que contribui como referência para a equipe multidisciplinar no atendimento do paciente ${ }^{13}$. Além disso, observa-se que a ausência de normas e de regras e protocolos não dependem somente de apoio tecnológico ${ }^{14}$. Para inserir essa prática recomenda-se a educação continuada da equipe e a disponibilização de registros digitais ou impressos que sejam acessados de maneira prática pelos profissionais ${ }^{13}$.

Um fato que merece destaque é a necessidade de planejamento da internação, alta hospitalar e gerenciamento de leitos da instituição estudada. Atualmente a instituição utiliza um sistema operacional SOUL MV, considerado líder em software de gestão em saúde. De acordo com a literatura, instituições que envolvem a gestão de leitos e possuem parceria com o referido sistema eletrônico gerem leitos de maneira satisfatória ${ }^{15}$, mas, para isso, a instituição deve trabalhar respeitando setores e especialidades. Nesse sentido, são necessárias linhas de cuidados específicos que tracem o perfil do paciente a ser atendido e invistam em pessoal para a estruturação deste serviço, de forma a facilitar a comunicação no processo de internação e alta hospitalar na instituição ${ }^{14,15}$. Essas iniciativas corroboram com a reflexão de que a importância do processo de internação hospitalar está relacionada à valorização do próximo como um ser humano ${ }^{16}$.

Sobre a comunicação entre a equipe multiprofissional e o paciente no pré-operatório, as falas dos entrevistados apontam que o quantitativo inadequado de funcionários para a demanda do serviço e o fluxo intenso de pacientes no setor contribuem para que a comunicação não seja efetiva. No sentido de minimizar essa lacuna, a literatura na narrativa de um trabalho em equipe identificou que o apoio mútuo, o respeito e trabalho em conjunto são pontos fortes encontrados no ambiente de trabalho para uma comunicação de qualidade ${ }^{17}$.

O lapidar da comunicação entre uma equipe multiprofissional depende de uma mudança necessária na cultura dos profissionais. Um dado interessante encontrado na literatura foi o destaque do aplicativo Whatsapp como ferramenta facilitadora nesse processo. $O$ aplicativo permite que os profissionais se reúnam virtualmente, formando um round com mensagens instantâneas, mais ágeis, solucionando dúvidas de uma forma geral ${ }^{18}$.

A comunicação multidisciplinar é considerada fator que influencia na segurança do paciente. Esta não é responsabilidade somente de uma categoria profissional, devendo ser preservada por toda a equipe multiprofissional ${ }^{19}$. 
Alude-se que a comunicação efetiva e a humanização, quando se fazem presentes, são o prisma, o aparato na prestação do cuidado, sendo ótica para a incontestabilidade da assistência à saúde, um feito intrínseco e necessário no processo de internação e do cuidar em saúde, necessitando de sociabilidade maior entre a tríade equipe de saúde-pacientefamiliar 20,21 .

Em relação à linha de cuidados prestados ao paciente pela equipe multiprofissional no período pré-operatório, diversas foram as falas sobre esses cuidados; dentre elas, abordagem sucinta do paciente como uma breve anamnese, aferição de sinais vitais, conciliação medicamentosa e orientações sobre o procedimento cirúrgico. Os cuidados básicos prestados ao paciente em geral podem ser simples, conforme os relatos na pesquisa, porém podem se tornar complexos quando avaliados conforme a especificidade de cada paciente. No entendimento literário, necessita-se de atenção especial não só do enfermeiro, como de toda a equipe multidisciplinar. Os cuidados básicos ofertados ao paciente estão relacionados às necessidades humanas de eliminação, sono, repouso, alimentação e promoção do conforto ${ }^{22,23}$

Com essa ótica, as principais recomendações e cuidados pré-operatórios são: jejum pré-operatório, atenção para a ansiedade e nível de estresse do paciente, a interrupção do tabagismo antes da cirurgia, a normotermia, controle glicêmico, a tricotomia no tempo ideal, a seleção da profilaxia antibiótica e o banho pré-operatório com clorexidina degermante ${ }^{22-24}$. Esses são cuidados pré-operatórios com intervenções centrais baseados em evidências científicas que contribuem para um bom prognóstico cirúrgico do paciente.

Neste sentido, torna-se necessário desenvolver uma sistematização do processo de internação do paciente para todos os membros da equipe multiprofissional que, de modo direto ou indireto, estejam inseridos nesse processo de forma centrada, todos caminhando em uma única direção, almejando um atendimento humanizado, com respaldo científico, ajustável e resolutivo ${ }^{19-22}$.

Foram consideradas limitações desse estudo a impossibilidade de aprofundamento teórico, devido às poucas publicações sobre a temática processo de internação hospitalar, além da grande quantidade de funcionários atuantes no cenário remanejados de outras clínicas, os quais não fazem parte do quadro do setor, impossibilitando-os de participarem da pesquisa.

\section{CONCLUSÃO}

Conclui-se que a falta de normas e protocolos articulados com ações e treinamentos que facilitem a equipe multiprofissional no atual processo de convocação de pacientes para o procedimento cirúrgico foi um dificultador desse processo. Além disso, a comunicação entre a equipe multiprofissional e o paciente foi prejudicada, visto que o grande fluxo de pacientes para procedimentos cirúrgicos e a não padronização dos serviços dificultaram as ações rotineiras dos profissionais. Na linha de cuidados prestados ao paciente pela equipe multiprofissional, os mais citados foram breve anamnese, aferição de sinais vitais, conciliação medicamentosa e orientações gerais.

Destaca-se a importância de ter uma assistência pautada em protocolos, pois, além de ser uma ferramenta eficaz, a sua utilização direciona profissionais de saúde, potencializa a qualidade do atendimento hospitalar e proporciona ao usuário um atendimento humanizado e resolutivo.

A pesquisa contribui para aumentar a produção científica sobre o tema, provocar reflexão na equipe multiprofissional de saúde, bem como incentivar futuras mudanças que beneficiem os pacientes cirúrgicos e profissionais de saúde. Este estudo é relevante e sugere inclusão de políticas que reduzam e promovam ajustes administrativos na rotina operacional hospitalar.

\section{REFERÊNCIAS}

1. Donabedian A. The seven pillars of quality. Arch. pathol. lab. med. [internet]. 1990 [cited 2019 Dec 10]; 114(11):1115-8. Avaiable from: https://www.nescon.medicina.ufmg.br/biblioteca/registro/The_seven_pillars_of_quality/367

2. Gonçalves KKN, Silva JI, Gomes ET, Pinheiro LLS, Figueiredo TR, Bezerra SMMS. Anxiety in the preoperative period of heart surgery. Rev. bras. enferm. [internet]. 2016 [cited 2018 May 24]; 69(2): 374-80. DOI: https://doi.org/10.1590/00347167.2016690225

3. Albuquerque NMQ, Cruz ICF. Guidelines for evidence-based practice on nursing prescription and management of hypovolemia in ICU: a systematized review. Journal of specialized nursing care [online]. 2018 [cited 2019May 20]; 10(1):1-9. Available from: http://www.jsncare.uff.br/index.php/jsncare/article/view/2991/761

4. Gonçalves $T$, Medeiros $V$. The preoperative visit as the anxiety mitigating factor in surgical patients. Revista SOBECC [internet]. 2016 [cited 2019 May 20]; 21(1):22-7. Available from: https://revista.sobecc.org.br/sobecc/article/view/38

5. Ministério da Saúde (Br). Diretrizes para a implantação de complexos reguladores. 2ª ed. Brasília: Ministério da Saúde, 2010. p. 41-50 [cited2018 Sep17]. Available from: http://bvsms.saude.gov.br/bvs/publicacoes/pacto_saude_volume6.pdf

6. Pinto LF, Soranz D, Scardua MT, Silva IM. Ambulatory municipal regulation of the Unified Health System services in Rio de Janeiro: advances, limitations and challenges. Ciênc. saúde colet. [internet]. 2017 [cited 2018 Aug 10]; 22(4):1257-67. DOI: http://dx.doi.org/10.1590/1413-81232017224.26422016. 
7. Oliveira AM, Soares $E$. The Communication as an educational tool during kidney transplantation preoperative period. Rev. pesqui. cuid. fundam. [online]. 2018 [cited 2018Sept 27]; 10(3):753-7. DOI: http://dx.doi.org/10.9789/21755361.2018.v10i3.753-757

8. Bohrer CD, Marques LGS, Vasconcelos RO, Oliveira JLC, Nicola AL, Kawamoto AM. Communication and patient safety culture in the hospital environment: vision of multiprofissional team. Rev. enferm. UFSM. [internet]. 2016 [cited 2019Sept 22]; 6(1):50-60. DOI: http://dx.doi.org/10.5902/2179769219260

9. Ministério da Saúde (Br). Conselho Nacional de Saúde. Comissão de Ética e Pesquisa. Resolução no 466/2012 sobre pesquisa envolvendo seres humanos. Brasília (DF): CNS; 2012.

10. Bardin L. Análise de conteúdo. Lisboa (Pt): Edições 70; 2011.

11. Oliveira DC. Análise de conteúdo temático-categorial: uma técnica maior nas pesquisas qualitativas. In: Lacerda MR, Ribeiro RP, Costenaro RGS, organizadoras. Metodologia da pesquisa para enfermagem e saúde da teoria à prática. 1a ed. Porto Alegre (PA): Moriá; 2018. p. 467-96.

12. Gomes ET, Galvão PCC, Santos KV, Bezerra SMMS. Risk factors for anxiety and depression in the preoperative period of cardiac surgery. Enferm. glob. [internet]. 2019 [cited 2019 Apr 01]; 18(2):426-69. DOI: https://doi.org/10.6018/eglobal.18.2.322041

13. Silva CF, Souza DM, Pedreira LC, Santos MR, Faustino TN. Perceptions of the multi-professional team on the implementation of palliative care in intensive care units. Ciênc. saúde colet. [internet]. 2013 [cited 2019 May 19]; 18(9):2597-604. DOI: http://dx.doi.org/10.1590/S1413-81232013000900014

14. Rafa C, Malik MA, Pinochet LHC. Análise das variáveis do ambiente interno no gerenciamento de leitos em organizações hospitalares privadas: aplicação do software. Revista de administração hospitalar e inovação em saúde [internet]. 2018 [cited 2019Aug 10]; 14(4):19-39. DOI: http://dx.doi.org/10.21450/rahis.v14i4.4427

15. Silva MS. Gestão da informação para o planejamento e controle da capacidade operacional do serviço hospitalar [Master thesis]. Maceió: Universidade Federal de Alagoas; 2019.

16. Rolim CLA. Hospital education: a matter of right. Actualidades investigativas eneducación [internet]. 2019 [cited 2019 Aug 03]; 19(1):1-18. DOI: http://dx.doi.org/10.15517/aie.v19i1.35600

17. Fassarella CS, Silva LD, Camerini FG, Figueiredo MCB. Organizational indicator of safety culture in a university hospital. Rev. enferm. UERJ [internet]. 2019 [cited 2019 Sep 13]; 27(spe):e34073. DOI: https://doi.org/10.12957/reuerj.2019.34073

18. Guzinski C, Lopes ANM, Flor J, Migliavaca J, Tortato C, Pai DD. Good practices for effective communication: the experience of the interdisciplinary round in orthopedic surgery. Rev. Gaúcha Enferm. [internet]. 2019 [cited 2019 Dec 20]; 40(spe):e2080353. DOI: http://dx.doi.org/10.1590/1983-1447.2019.20180353

19. Henriques AHB, Costa SS, Lacerda JS. Nursing care in surgical patient safety: an integrative review. Cogitare enferm. [internet]. 2016 [cited 2019 Sep 22]; 21(4):01-09. DOI: http://dx.doi.org/10.5380/ce.v21i4.45622

20. Piexak DR, Ferreira CLL, Terra MG, Backes DS, Barlem JGT, Ilha S. Nursing care in surgical inpatient unit: perception of patients. Rev. pesqui. cuid. fundam. online. 2016 [cited 2019 Sep 22]; 8(1):3624-32. DOI: http://dx.doi.org/10.9789/21755361.2016.v8i1.3624-3632

21. Lemos DMP, Barcellos RA, Borba DSM, Caballero LG, Goldraich LA, Echer IC. Effective communcation for the safe care of patients with ventricular assist device. Rev. Gaúcha Enferm. [internet]. 2019 [cited 2019Aug 19]; 40(esp):e20180344. DOI http://dx.doi.org/10.1590/1983-1447.2019.20180344

22. Rothrock JCA. Cuidados de enfermagem ao paciente cirúrgico. 13a ed. Rio de Janeiro (RJ): Elsevier; 2018.

23. Garcez JS, Sousa LCB, Novais Neta MB, Maia FL, Araújo FPC. Main recommendations in preoperative care. Rev. med. UFC [internet]. 2018 [cited 2019 Aug 05]; 59(1):53-60. DOI: http://periodicos.ufc.br/revistademedicinadaufc/article/download/32418/97079/

24. Ferraz ÁAB, Vasconcelos CFM, Santa-Cruz F, Aquino MAR, Buenos-Aires VG, Siqueira LT. Surgical site infection in bariatric surgery: results of a care bundle. Rev. col. bras. cir. [internet]. 2019 [cited 2019 Nov 03]; 46(4):e2252. DOI: http://dx.doi.org/10.1590/0100-6991e-20192252 